\title{
Prognostic factors for recurrence after bilateral rectus recession procedure in patients with intermittent exotropia
}

Eye (2012) 26, 899; doi:10.1038/eye.2012.67

Correction to: Eye (2012) 26, 846-852;

doi:10.1038/eye.2012.55; published online 23 March 2012

Since the publication of the above article, the authors have noticed that the number of

Table 2 Demographic characteristics of patients with intermittent exotropia

\begin{tabular}{|c|c|}
\hline Characteristic & No. of patients (\%) \\
\hline Gender (male: female) & $240: 271$ \\
\hline Photophobia (presense: absence) & $351: 160$ \\
\hline Diplopia (presense: absence) & 10:501 \\
\hline Age of onset (mean $\pm S D$, years) & $3.01 \pm 2.09$ \\
\hline Age at operation (mean $\pm S D$, years) & $6.34 \pm 1.86$ \\
\hline Dcc at pre-op $($ mean $\pm S D, P D)$ & $21.95 \pm 2.92$ \\
\hline \multicolumn{2}{|l|}{ SE refractive error $(D)^{\mathrm{a}}$} \\
\hline$<-4.00$ & 7 \\
\hline$-4.00 \sim<-3.00$ & 5 \\
\hline$-3.00 \sim<-2.00$ & 19 \\
\hline$-2.00 \sim<-1.00$ & 55 \\
\hline$-1.00 \sim<0.00$ & 110 \\
\hline$+000 \sim<+1.00$ & 221 \\
\hline$+1.00 \sim<+2.00$ & 79 \\
\hline$\geq+2.00$ & 15 \\
\hline \multicolumn{2}{|l|}{$\operatorname{Astigmatism}(D)^{\mathrm{b}}$} \\
\hline$<0.50$ & 278 \\
\hline $0.50 \sim<1.00$ & 104 \\
\hline $1.00 \sim<1.50$ & 49 \\
\hline $1.50 \sim<2.50$ & 59 \\
\hline$\geq 2.50$ & 21 \\
\hline \multicolumn{2}{|l|}{ SE anisometropia (D) } \\
\hline$<0.50$ & 434 \\
\hline $0.50 \sim<1.00$ & 59 \\
\hline$\geq 1.00$ & 18 \\
\hline
\end{tabular}

Abbreviations: D, diopters; Dcc, distance deviation with correction; PD, prism diopter; $\mathrm{SE}$, spherical equivalent. ${ }^{a}$ Level of refractive error defined by the less hyperopic eye for SE refractive error.

${ }^{b}$ Level of refractive error defined by the less astigmatic eye for astigmatic refractive error.
SH Lim, BS Hwang and MM Kim

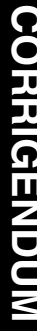

patients with an SE refractive error of -3.00 $\sim<-2.00$ in Table 2 was incorrect. The correct table is shown below.

The authors would like to apologize for this mistake. 